Strand, K. Aa. The orbital motion of 6I Cygni.

An examination of the orbital motion in 61 Cygni, from 232 multiple-exposure photographic plates taken with the long-focus refractors at Potsdam, Lick, Sproul, Yerkes, Lowell and Dearborn Observatories, confirms the perturbation previously announced (Strand 1943), which was based on part of the present material, 47 plates. While the early plates in the series cover the years 1914-I8, the majority of the plates were taken between 1937 and 1955, during which time plates were obtained at each opposition except for the years 1938 and 1944 .

The new investigation leads to a circular orbit with $a=$ ".o102 \pm ".o012 (m.e.) and a period of 4.8 years against the previously found period of 4.9 years.

With 720 years for the period of the large orbit, $a=24$ ".616, and the parallax ".294, the total mass of the system is I.I $\odot \odot$ with $M_{A}=.58$, $\mathrm{M}_{\mathrm{B}}=.54$ and $\mathrm{M}_{\mathrm{C}}=.008$. The mass of Component $\mathrm{C}$ has been derived under the assumption that it contributes no light to the observed motion.

Strand, K. Aa. 1943, Proc. Amer. Phil. Soc. 86, 364.

Dearborn Observatory, Evanston, Ill.

\section{Struve, Otto. High dispersion observations of Spica.}

Spectrographic observations of Spica with a dispersion about $4.5 \AA \mathrm{A} / \mathrm{mm}$ have been recently secured at the Mount Wilson Observatory on Process emulsion. Striking changes in profile and structure of the spectral lines have been found, and these changes do not repeat themselves in different cycles. Structure is present at all phases in the lines of the stronger component of the system, and it is specially prominent when the star is approaching. The period and the origin of phases derived at the Yerkes Observatory twenty-two years ago were adequate to represent the new observations.

Berkeley Astronomical Department, University of California, Berkeley, Calif.

\section{Vasilevskis, S. Automatic measurement of astro-} graphic plates.

The demand for accurate stellar proper motions steadily increases, but the number of active workers in astrometry decreases. Thus there is a possibility of dangerous delay in completing several major astrometric programs in progress or planning, if the approach to routine work in astrometry does not fundamentally change. The measurement of astrographic plates is a major part of this routine work.

The pioneering in new measuring technique has been done by the Watson Scientific Computing Laboratory of I.B.M. (Lentz and Bennet 1954). The Lick Observatory, in cooperation with the Farrand Controls, Inc., New York, is planning a new automatic measuring machine for the Lick Proper Motion Program (Vasilevskis I954), and for the probable extension of a similar program to the southern sky.

In the proposed design a viewing frame is used to survey a plate pair by blinking, to select the objects for measurement, and to record on punchcards the approximate rectangular coordinates of objects selected. The approximate coordinates are used as input data for automatic guiding of a photoelectric scanner to the positions of the objects in the precise measuring engine. The machine is equipped with two perpendicular precise scales, Inductosyns, developed by the Farrand Controls. The Inductosyn and a slider comprise two circuit elements, and the motion of the slider along the scale is transformed into a digital record, the sensitivity being 0.25 micron. As soon as the scanner "sees" the image, it centers automatically on the image, and the position of the scanner is automatically recorded in both rectangular coordinates on a punch-card.

Lentz, J. and Bennet, R. 1954, Electronics 27, No. 6, I 58 . Vasilevskis, S. 1954, A. J. 59, 40. Lick Observatory, University of California, Mt. Hamilton, Calif.

Velghe, Albert G. Low dispersion spectra in the yellow-red region, and their usefulness in some distribution problems.

In connection with a survey of $H \alpha$ emission objects in selected fields of the southern Milky Way, photographic plates were taken with the $3^{\circ}$ objective prism (32-inch diameter) attached to the ADH Baker-Schmidt telescope of the Boyden Station. Eastman Kodak Ioza-E plates were exposed through a yellow filter, Wratten No. I5. This combination yields spectra covering the region $\lambda \lambda_{5} 100-6700$. On normally exposed images, however, only the features within the spectral range $\lambda \lambda 5400-6600$ are clearly shown. The length of the spectra is about $2 \mathrm{~mm}$ and the dispersions are $450 \AA / \mathrm{mm}$ at $\lambda 5500$ and $900 \AA$ / $\mathrm{mm}$ at $\lambda 6500$. Since the primary purpose of the 